\title{
Deletions, extensions, and reductions of elliptic semiplanes
}

\author{
Marien Abreu Martin Funk Domenico Labbate \\ Vito Napolitano*
}

\begin{abstract}
We present three constructions which transform some symmetric configuration $\mathcal{K}$ of type $n_{k}$ into new symmetric configurations of types $(n+1)_{k}$, or $n_{k-1}$, or $((\lambda-1) \mu)_{k-1}$ if $n=\lambda \mu$. Applying them to Desarguesian elliptic semiplanes, an infinite family of new configurations comes into being, whose types fill large gaps in the parameter spectrum of symmetric configurations.
\end{abstract}

Keywords : configurations, elliptic semiplanes, 1-factors, Martinetti extensions MSC 2000: 05B30

\section{The parameter spectrum of configurations of type $n_{k}$}

For notions from incidence geometry and graph theory, we refer to [10] and [7], respectively.

A (tactical) configuration of type $\left(n_{r}, b_{k}\right)$ is a finite incidence structure consisting of a set of $n$ points and a set of $b$ lines such that (i) each line is incident with exactly $k$ points and each point is incident with exactly $r$ lines, (ii) two distinct points are incident with at most one line. If $n=b$ (or equivalently $r=k$ ), the configuration is called symmetric and its type is indicated by the symbol $n_{k}$.

The deficiency of a symmetric configuration $\mathcal{C}$ is $d:=n-k^{2}+k-1$. The deficiency is zero if and only if $\mathcal{C}$ is a finite projective plane.

\footnotetext{
*This research was carried out by M. Abreu, D. Labbate and V. Napolitano within the activity of INdAM-GNSAGA and supported by the Italian Ministry MIUR.
} 


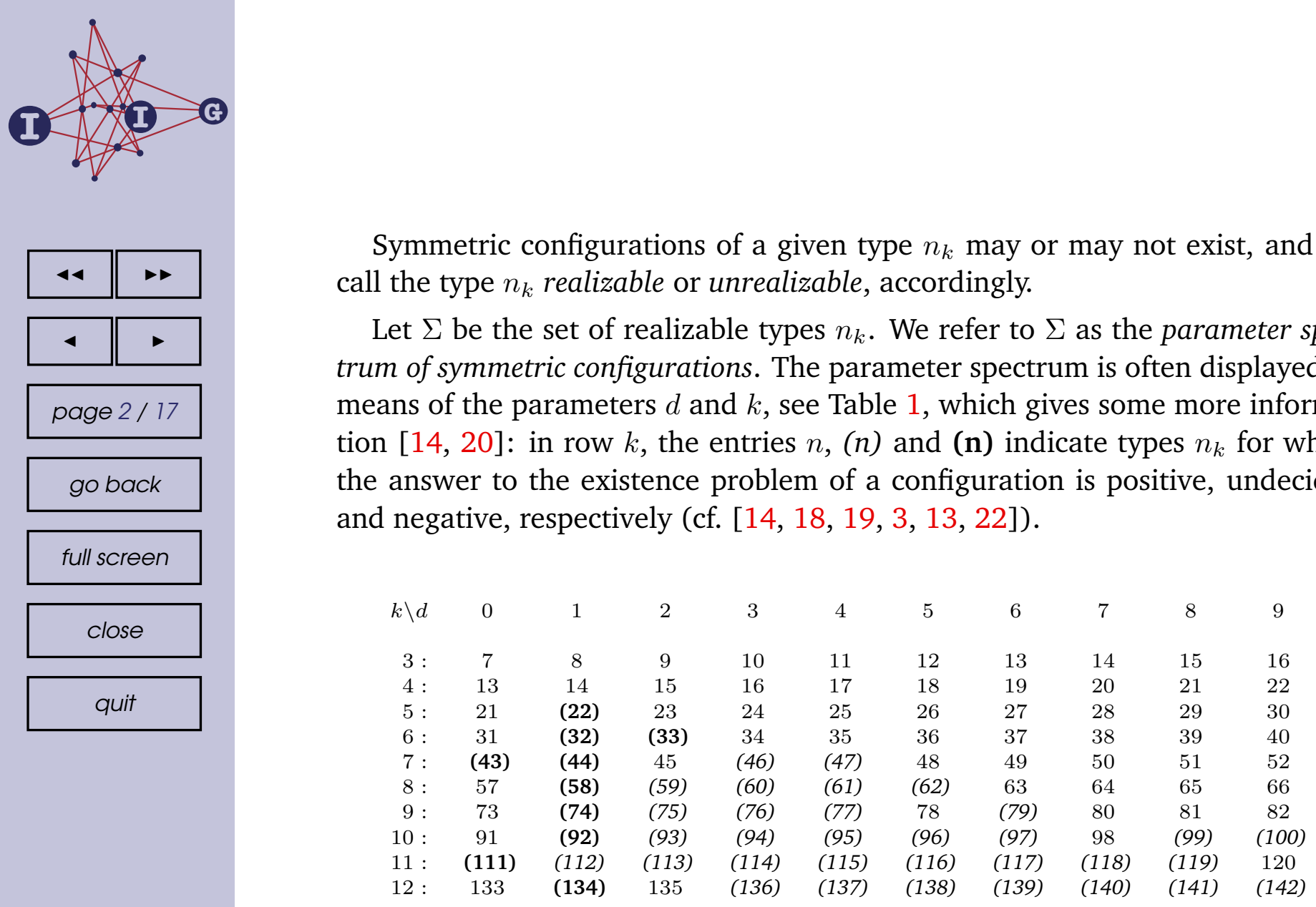

Table 1: The parameter spectrum of symmetric configurations

In the lower left triangle of $\Sigma$, the existence of instances is highly in doubt. As far as they exist, elliptic semiplanes dominate the region. Recall that an elliptic semiplane of order $\nu$ is a configuration of type $n_{\nu+1}$ satisfying the following axiom of parallels: given a non-incident point-line pair $(p, l)$, there exists at most one line $l^{\prime}$ through $p$ parallel to $l$ (i.e. $l$ and $l^{\prime}$ are not concurrent) and at most one point $p^{\prime}$ on $l$ parallel to $p$ (i.e. $p$ and $p^{\prime}$ are not collinear). Dembowski [10] provided a classification of elliptic semiplanes in types called $O, C, L, D$ and $B$, which we will use in the sequel.

Consider any finite projective plane of order $n$. An anti-flag is a non-incident point-line pair $(p, l)$. The pencil (of lines) through a point is the set of lines that are incident with that point. By removing from a projective plane $\mathcal{P}$ an antiflag $(p, l)$ as well as the pencil through $p$ and all the points on $l$, we obtain an elliptic semiplane $\mathcal{L}$ of type $L$ [10] which is a configuration of type $\left(q^{2}-1\right)_{q}$ and deficiency $q-2$. Since projective planes of order $q$ exist for each prime-power $q$, this construction furnishes an infinite family of configurations of type $\left(q^{2}-1\right)_{q}$. If $\mathcal{P}=\mathrm{PG}(2, q)$ is Desarguesian, the corresponding Desarguesian semiplane of type $L$ will be denoted by $\mathcal{L}_{q}$. We call them the anti-flag examples. They lie in the second upper diagonal of $\Sigma$ (called anti-flag diagonal).

A flag of a projective plane of order $n$ is a point-line pair $(p, l)$ with $p \mid l$. By removing from a projective plane $\mathcal{P}$ a flag $(p, l)$ as well as the pencil through $p$ 



\section{$1 \frac{1}{1}=0$ \\ non-isomorphic configurations, dual to each other.}
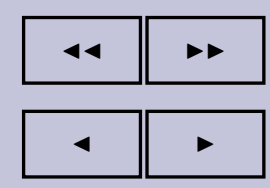

page $5 / 77$

go back

full screen

close

quit

ACADEMIA

PRESS

WW

$\widehat{\underline{\text { IIIIII }}}$
A corollary to the famous Marriage Theorem by Ph. Hall [16] states: every $k$-regular bipartite graph $\Lambda$ is 1-factorable (cf. e.g. [17, Theorem 3.2]). This implies that the edge set $E(\Lambda)$ can be partitioned into a union of $k$ pairwise disjoint 1 -factors $F_{i}, i=1, \ldots, k$.

Let $\Lambda$ be the Levi graph of some configuration $\mathcal{K}$ of type $n_{k}$ and choose a 1 -factor $F_{i}$ of $\Lambda$, for some $i \in\{1, \ldots, k\}$. Let $\Lambda^{(1 F)}$ be the subgraph of $\Lambda$ with vertex set $V\left(\Lambda^{(1 F)}\right)=V(\Lambda)$ and edge set $E\left(\Lambda^{(1 F)}\right)=E(\Lambda) \backslash E\left(F_{i}\right)$. Obviously, $\Lambda^{(1 F)}$ is a $(k-1)$-regular bipartite graph on $2 n$ vertices, which can be seen as the Levi graph of some configuration of type $n_{k-1}$. Since we are only interested in its type $n_{k-1}$ being realizable, any such configuration will be denoted by $\mathcal{K}^{(1 F)}$ and referred to as a configuration obtained from $\mathcal{K}$ by a 1 -factor deletion.

This construction can be reiterated $\nu$ times for some $\nu \in\{1, \ldots, k-3\}$, for pairwise distinct 1-factors belonging to a fixed 1-factorisation of $\Lambda$. We denote the resulting configuration by $\mathcal{K}^{(\nu F)}$.

If we embed the parameter spectrum of symmetric configurations $\Sigma$ into $\mathbb{R}^{2}$, the realizable types $n_{k}, n_{k-1}, \ldots, n_{3}$ lie on a parabola since, for fixed $n$ and $k$, the deficiency of the type $n_{k-\nu}$ seen as a function of $\nu=0, \ldots, k-3$ reads

$$
d(k-\nu)=-\nu^{2}+(2 k-1) \nu+d(k)
$$

where $d(k)=n-k^{2}+k-1$ is the deficiency of $\mathcal{K}$ and does not depend on $\nu$. The vertex of the parabola is the point $\left(\frac{1}{2},\left(k-\frac{1}{2}\right)^{2}+d(k)\right)$, which lies outside of $\Sigma$. Hence distinct types out of $\left\{n_{k}, n_{k-1}, \ldots, n_{3}\right\}$ have distinct deficiencies.

\section{Parallel flags in configurations and Martinetti extensions}

Two distinct points (lines) of a configuration $\mathcal{K}=(P, L, \mid)$ are said to be parallel if there is no line (point) incident with both of them. We extend this concept and call two flags $\left(p_{1}, l_{1}\right)$ and $\left(p_{2}, l_{2}\right)$, such that $p_{1} \neq p_{2}$ and $l_{1} \neq l_{2}$, parallel if both $\left\{p_{1}, p_{2}\right\}$ and $\left\{l_{1}, l_{2}\right\}$ make up pairs of parallel elements. A family of pairwise parallel flags in a configuration of type $n_{k}$ is said to be a hyperpencil if it has cardinality $k-1$.

Definition 3.1. Let $\mathcal{K}=(P, L, \mid)$ be a configuration of type $n_{k}$ and

$$
\mathcal{H}=\left\{\left(p_{i}, l_{i}\right): p_{i} \mid l_{i} \text { for } i=1, \ldots, k-1\right\}
$$

a hyperpencil of parallel flags in $\mathcal{K}$. Then the Martinetti extension $\mathcal{K}_{\mathcal{H}}$ of $\mathcal{K}$ is the incidence structure obtained from $\mathcal{K}$ by 


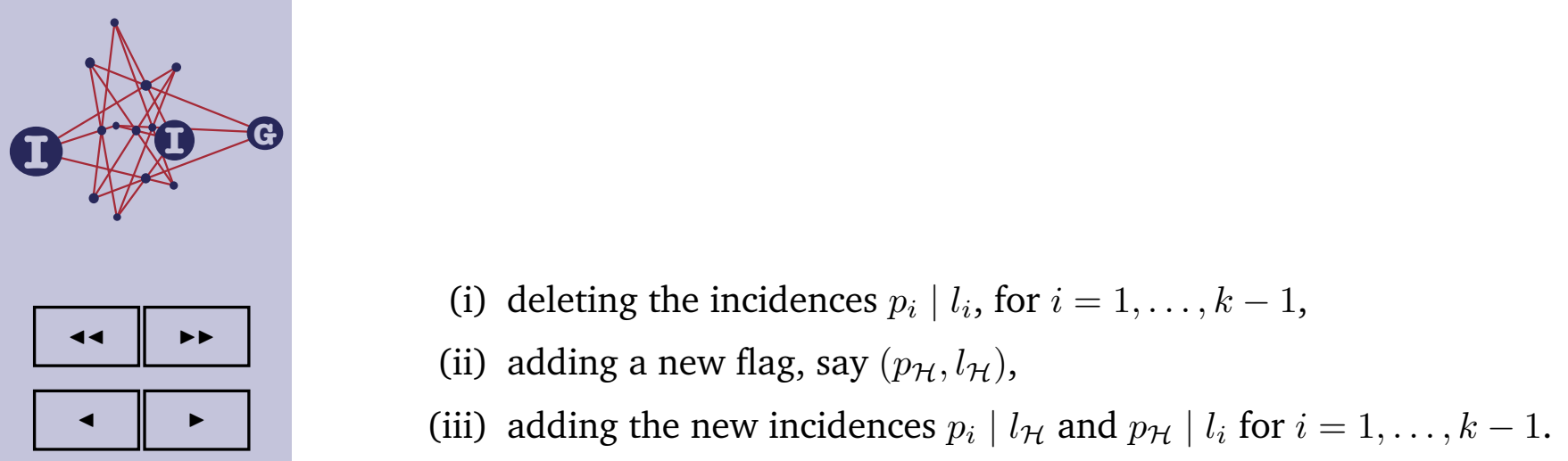

page $6 / 17$

go back

full screen

close

quit
Remark 3.2. The case $k=3$ has already been pointed out by Martinetti [21].

The following is a special case of [11, Proposition 2.5].

Proposition 3.3. If $\mathcal{K}$ is a configuration of type $n_{k}$, then $\mathcal{K}_{\mathcal{H}}$ is a configurations of type $(n+1)_{k}$.

Given a configuration $\mathcal{K}$ of type $n_{k}$ with a suitable hyperpencil of parallel flags, we are only interested in the existence of Martinetti extensions of $\mathcal{K}$ as configurations having realizable type $(n+1)_{k}$. Therefore any such configuration will be denoted by $\mathcal{K}^{(1 M)}$.

Next we investigate the possibilities to iterate this construction.

Definition 3.4. Let $\mathcal{K}$ be a configuration of type $n_{k}$. Two hyperpencils

$$
\begin{aligned}
\mathcal{F} & =\left\{\left(r_{i}, l_{i}\right): r_{i} \mid l_{i} \text { for } i=1, \ldots, k-1\right\} \text { and } \\
\mathcal{G} & =\left\{\left(s_{i}, m_{i}\right): s_{i} \mid m_{i} \text { for } i=1, \ldots, k-1\right\}
\end{aligned}
$$

of parallel flags are disjoint if all involved elements $r_{i}, s_{i}$ and $l_{i}, m_{i}$ are distinct in pairs.

Corollary 3.5. Let $\mathcal{K}$ be a configuration of type $n_{k}$ and $\mathcal{F}, \mathcal{G}$ be two disjoint hyperpencils of parallel flags. Then $\left(\mathcal{K}_{\mathcal{F}}\right)_{\mathcal{G}}$ is isomorphic to $\left(\mathcal{K}_{\mathcal{G}}\right)_{\mathcal{F}}$ and is of type $(n+2)_{k}$.

Proof. It is enough to apply [11, Proposition 2.5].

Accordingly, any configuration obtained from a configuration $\mathcal{K}$ of type $n_{k}$ by $\nu$ Martinetti extensions will be denoted by $\mathcal{K}^{(\nu M)}$.

\section{Reducing polysymmetric configurations}

Let $A$ be a square $(0,1)$-matrix. We call $A$ doubly $k$-stochastic if there are $k$ entries 1 in each row and column. Recall that, with each permutation $\pi$ in the symmetric group $\mathcal{S}_{\mu}$, we can associate its permutation matrix $P_{\pi}=\left(p_{i j}\right)_{1 \leq i, j \leq \mu}$ which is defined by $p_{i j}=1$ if $\pi(i)=j$, and $p_{i j}=0$ otherwise. Distinct permutations $\pi, \rho \in S_{\mu}$ (as well as the corresponding permutation matrices $P_{\pi}$ and 







\section{Applications and open problems}

Applying Theorems 6.2, 6.3, and 6.4, we compute all the new realizable configuration types obtained from elliptic semiplanes within region $\Delta$ of Figure 1 . For each $\alpha \in\{0, \ldots, q-3\}, \beta \in\{0, \ldots, q-\alpha\}$, and $\gamma \in\{0, \ldots, q-\alpha-3\}$, Theorems 6.2 and 6.3 imply that the configurations types $n_{k}=\left(q^{2}-\alpha q+\right.$ $\beta)_{q-\alpha-\gamma}$ and $n_{k}=((q+1-\alpha)(q-1)+\beta)_{q-\alpha-\gamma}$ are realizable. The types $133_{11}, 183_{13}, 307_{17}, 381_{19}, 553_{23}$ are realizable as a 1 -factor deletion $\mathcal{P}_{q}^{(1 F)}$ of the finite Desarguesian projective plane $\mathcal{P}_{q}$ with $q=11,13,17,19,23$. Theorem 6.4 and the explicit representation of $\mathcal{P}_{q^{2}}$ (see Section 5) support the following types:

$$
\begin{array}{llll}
231_{15}, 210_{14}, 189_{13} & : & \mathcal{P}_{16}^{((\nu+1) R)} & \text { for } \nu=1,2,3 \\
589_{24}, 558_{23}, 434_{19}, 403_{18} & : & \mathcal{P}_{25}^{((\nu+1) R)} & \text { for } \nu=1,2,6,7
\end{array}
$$

For $7 \leq k \leq 25$, the types lying in $\Delta$ that become realizable through our methods are listed in the following table:

\begin{tabular}{r|c|l|c}
$k$ & $k^{2}-1$ & intervals of realizable types $n_{k}$ & $\left(2 l_{k}+1\right)_{k}$ \\
\hline 7 & 48 & $48_{7} \ldots 50_{7}$ & $51_{7}$ \\
8 & 63 & $63_{8} \ldots 68_{8}$ & $69_{8}$ \\
9 & 80 & $80_{9} \ldots 88_{9}$ & $89_{9}$ \\
10 & 99 & $110_{10}$ & $111_{10}$ \\
11 & 120 & $120_{11} \ldots 133_{11}$ & $145_{11}$ \\
12 & 143 & $156_{12} \ldots 170_{12}$ & $171_{12}$ \\
13 & 168 & $168_{13} \ldots 183_{13} ; 189_{13} ; 208_{13} \ldots 212_{13}$ & $213_{13}$ \\
14 & 195 & $210_{14} ; 224_{14} \ldots 254_{14}$ & $255_{14}$ \\
15 & 224 & $231_{15} ; 240_{15} \ldots 302_{15}$ & $303_{15}$ \\
16 & 255 & $255_{16} \ldots 354_{16}$ & $355_{16}$ \\
17 & 288 & $288_{17} \ldots 307_{17} ; 323_{17} \ldots 380_{17} ; 391_{17} \ldots 398_{17} \ldots$ & $399_{17}$ \\
18 & 323 & $342_{18} \ldots 380_{18} ; 403_{18} ; 414_{18} \ldots 432_{18}$ & $433_{18}$ \\
19 & 360 & $360_{19} \ldots 381_{19} ; 434_{19} ; 437_{19} \ldots 492_{19}$ & $493_{19}$ \\
20 & 399 & $460_{20} \ldots 566_{20}$ & $567_{20}$ \\
21 & 440 & $483_{21} \ldots 666_{21}$ & $667_{21}$ \\
22 & 483 & $506_{22} \ldots 712_{22}$ & $713_{22}$ \\
23 & 528 & $528_{23} \ldots 553_{23} ; 558_{23} ; 575_{23} \ldots 744_{23}$ & $745_{23}$ \\
24 & 575 & $589_{24} ; 600_{24} \ldots 850_{24}$ & $851_{24}$ \\
25 & 624 & $624_{25} \ldots 650_{25} ; 675_{25} \ldots 960_{25}$ & $961_{25}$
\end{tabular}

Table 2: Realizable types for $7 \leq k \leq 25$ obtained through our methods

Funk has found configurations of types $107_{10}, 108_{10}, 109_{10}, 110_{10}$ through a computer search using cyclic difference sets [12]. Performing further computer searches on cyclic difference sets we have found the following configurations:

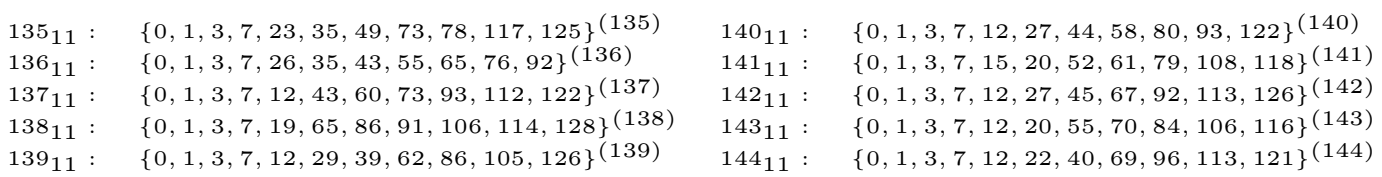




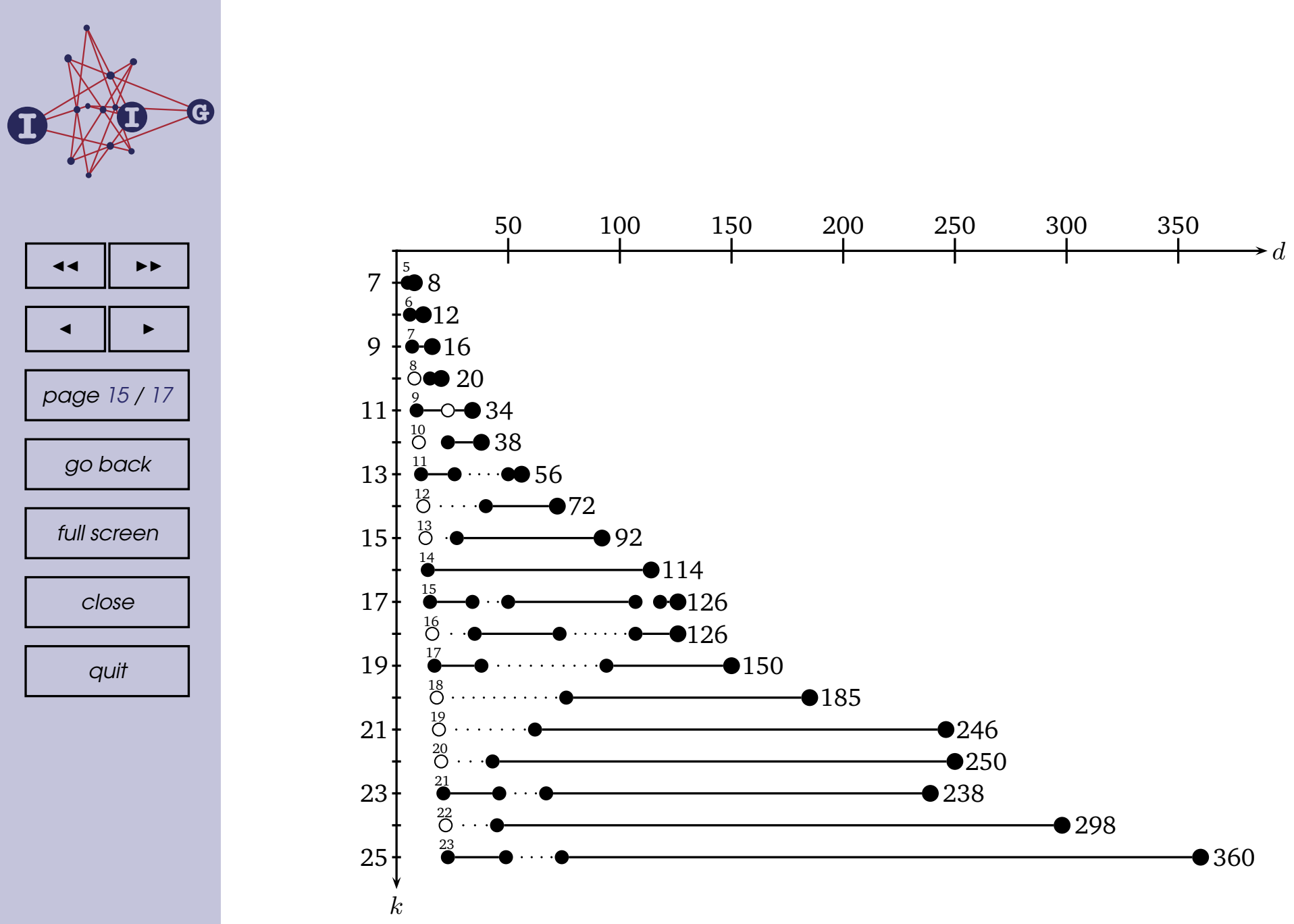

Figure 2: Region $\Delta$ including our new results

[6] G. S. Bloom, S. W. Golomb, in Numbered Complete Graphs, Unusual Rulers, and Assorted Applications. Theory and Applications of Graphs (Proc. Internat. Conf., Western Mich. Univ., Kalamazoo, Mich., 1976), Lecture Notes in Math. 642, Springer, Berlin-Heidelberg-New York (1978), pp. 53-65.

[7] J. A. Bondy and U. S. R. Murty, Graph Theory with Applications, North Holland, New York-Amsterdam-London, 1976.

[8] H. S. M. Coxeter, Self-dual configurations and regular graphs, Bull. Amer. Math. Soc. 56 (1950), 413-455.

[9] P. J. Davis, Circulant Matrices, Chelsea Publ., New York, 1994.

[10] P. Dembowski, Finite Geometries, Springer, Berlin-Heidelberg-New York, 1968 (reprint 1997).

[11] M. Funk, On configurations of type $n_{k}$ with constant degree of irreducibility, J. Combin Theory Ser. A 65 (1994), 173-201. 

, Cyclic difference sets of positive deficiency, Bull. Inst. Combin.

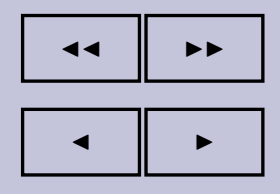

page $16 / 77$

go back

full screen

close quit

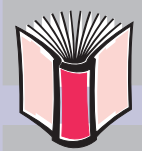

$\widehat{\underline{\text { IIIIII }}}$
[13] M. Funk, D. Labbate and V. Napolitano, Tactical (de)compositions of symmetric configurations, Discrete Math. 309 (2009), 741-747.

[14] H. Gropp, On the existence and non-existence of configurations $n_{k}, J$. Comb. Inf. Syst. Sci. 15 (1990), 34-48.

[15] _ Configurations and graphs, Discrete Math. 111 (1993), 269-276.

[16] Ph. Hall, On representatives of subsets, J. London Math. Soc. 10 (1935), 26-30.

[17] D. A. Holton and J. Sheehan, The Petersen Graph, Cambridge Univ. Press, Cambridge, 1993.

[18] P. Kaski and P. R. J. Östergård, There exists no symmetric configuration with 33 points and line size 6, Australasian J. Combin. 38 (2007), 273-277.

[19] V. Krčadinac, Construction and Classification of Finite Structures by Computer (in Croatian), Ph.D. Thesis, University of Zagreb, 2004.

[20] M. J. Lipman, The existence of small tactical configurations, in Graphs and Combinatorics (R. A. Bari, F. Harary, eds), Lecture Notes in Math. 406, Springer, Berlin-Heidelberg-New York (1974), pp. 319-324.

[21] V. Martinetti, Sulle configurazioni piane $\mu_{3}$, Ann. Mat. 15 (1887), 1-26.

[22] R. Mathon, Talk at the British Combinatorial Conference, 1987.

[23] N. S. Mendelsohn, P. Padmanabhan and B. Wolk, Planar projective configurations I, Note Mat. 7 (1987), 91-112.

[24] J. B. Shearer, IBM Personal communication, http://researchweb. watson. ibm. com/people/s/shearer/grtab.html. 

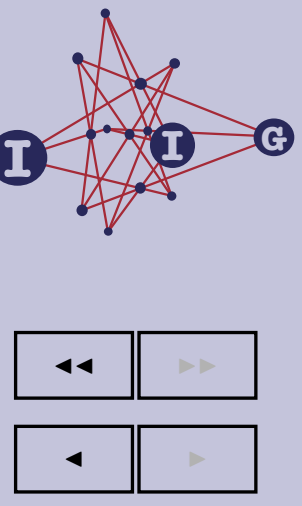

page $17 / 77$

go back

full screen

close

quit

\section{Domenico Labbate}

Dipartimento di Matematica, Politecnico di Bari, Via E. Orabona, 4, I-70125 Bari, Italy.

e-mail: labbate@poliba.it

Vito Napolitano

Dipartimento di IngegneRia Civile, Facoltà di IngegneRia, Seconda Università degli StUdi Di NAPOli, ReAl CASA DElL'ANNunziata, Via Roma, 29, I-81031 Aversa (CE), ItAly.

e-mail: vito.napolitano@unina2.it 